\title{
Modules de plus haut poids unitarisables sur la super-algèbre de Virasoro $N=2$ tordue
}

\author{
Kenji Iohara
}

April 12, 2006

\author{
Department of Mathematics, Faculty of Science, Kobe University, Kobe 657-8501, \\ Japan \\ e-mail : iohara@math.kobe-u.ac.jp
}

\section{Résumé}

Dans cet article, on classifie les modules de plus haut poids unitarisables sur la super-algèbre de Virasoro $N=2$ tordue.

\section{Introduction}

En 1984, les physiciens D. Friedan, Z. Qiu et S. Shenker ont énoncé une condition nécessaire d'unitarisabilité des modules de Verma sur l'algèbre de Virasoro [FQS1]. Ils ont fait la même recherche pour les super-algèbres de Virasoro $N=1$ [FQS2] en 1985. En 1986, les physiciens W. Boucher, D. Friedan et A. Kent [BFK] ont énoncé un théorème analogue pour les super-algèbres de Virasoro $N=2$, incluant le cas du secteur tordu. Tous les travaux mentionés ci-dessus sont sans démonstration.

R. Langlands [L] a démontré le théorème énoncé par [FQS1] en 1988. Suivant les idées de [L], F. Sauvageot a démontré celui de [FQS2] en 1989.

En 1986, les physiciens P. Goddard, A. Kent et D. Olive [GKO] ont construit les séries minimales unitarisables pour l'algèbre de Virasoro et les super-algèbres de Virasoro $N=1$ via la construction de commutant. Grâce à ce résultat, on sait que les conditions présentées dans [FQS1] et [FQS2] sont aussi des conditions suffisantes.

Le but de cet article est de démontrer le théorème de [BFK] pour la superalgèbre de Virasoro $N=2$ tordue. De plus, on démontre que la condition de [BFK] est aussi suffisante. En fait, on construit les séries minimales unitarisables de cette algèbre en considérant la version tordue de $[\mathrm{ZF}]$.

Dans la section 2, on rappelle la définition de la super-algèbre de Virasoro $N=2$ tordue et du module de Verma. Ensuite, on classifie les antiinvolutions anti-linéaires de cette algèbre. On rappelle aussi la définition d'une certaine forme hermitienne sur les modules de Verma, et finalement, on formule le théorème principal de cet article. Dans la section 3, on construit les séries 
minimales unitarisables explicitement. Après avoir rappelé les résultats concernant les formules de déterminants dans la section 4, on démontre le théorème principal dans la section 5 .

L'auteur est soutenu partiellement par JSPS Grant in Aid for Scientific Research. Une partie de cette recherche a été faite pendant mon séjour à KIAS, Korea Institute for Advanced Study. Je tiens à remercier cet institut pour son soutien et ses membres pour leur hospitalité. Je tiens à remercier aussi Y. Koga pour des discussions utiles.

\section{Le théorème principal}

Dans cette section, on rappelle les définitions de la super-algèbre de Virasoro $N=2$ tordue et de modules de types de Verma. Après la classification de ses anti-involutions anti-linéaires, on énonce le théorème principal de cet article.

\subsection{La Super-Algèbre de Virasoro $N=2$ Tordue}

Définition 2.1. La Super-Algèbre de Virasoro $N=2$ Tordue est la superalgèbre de Lie

$$
\mathfrak{g}:=\bigoplus_{n \in \mathbb{Z}} \mathbb{C} L_{n} \oplus \bigoplus_{m \in \frac{1}{2}+\mathbb{Z}} \mathbb{C} I_{m} \oplus \bigoplus_{i=1}^{2} \bigoplus_{k_{i} \in \frac{1}{2} i+\mathbb{Z}} \mathbb{C} G_{k_{i}}^{i} \oplus \mathbb{C} c
$$

avec la parité

$$
\operatorname{deg} L_{n}=\operatorname{deg} I_{m}=\operatorname{deg} c=\overline{0}, \quad \operatorname{deg} G_{k_{i}}^{i}=\overline{1},
$$

avec les relations

$$
\begin{aligned}
& {\left[L_{m}, L_{n}\right]=(m-n) L_{m+n}+\frac{1}{12}\left(m^{3}-m\right) \delta_{m+n, 0} c} \\
& {\left[L_{m}, G_{n}^{i}\right]=\left(\frac{m}{2}-n\right) G_{m+n}^{i}} \\
& {\left[G_{m}^{i}, G_{n}^{j}\right]=2 \delta_{i, j} L_{m+n}+\sqrt{-1}(m-n) \varepsilon_{i, j} I_{m+n}+\frac{1}{3}\left(m^{2}-\frac{1}{4}\right) \delta_{i, j} \delta_{m+n, 0} c} \\
& {\left[I_{m}, I_{n}\right]=\frac{1}{3} m \delta_{m+n, 0} c} \\
& {\left[I_{m}, G_{n}^{i}\right]=\sqrt{-1} \sum_{j=1}^{2} \varepsilon_{i, j} G_{m+n}^{j}} \\
& {\left[I_{m}, L_{n}\right]=m I_{m+n}}
\end{aligned}
$$

où $\delta_{i, j}$ signifie le symbole de Kronecker et l'on a posé

$$
\left(\varepsilon_{i, j}\right)_{1 \leq i, j \leq 2}=\left(\begin{array}{cc}
0 & 1 \\
-1 & 0
\end{array}\right)
$$


Remarque 2.1. On utilisera aussi les générateurs

$$
G_{m}^{ \pm}:=\frac{1}{\sqrt{2}} \times \begin{cases}G_{m}^{1} & m \in \frac{1}{2}+\mathbb{Z} \\ \pm \sqrt{-1} G_{m}^{2} & m \in \mathbb{Z} .\end{cases}
$$

Ils satisfont les relations suivantes: Pour $\varepsilon, \varepsilon^{\prime} \in\{ \pm\}$,

$$
\begin{aligned}
& {\left[L_{m}, G_{n}^{ \pm}\right]=\left(\frac{m}{2}-n\right) G_{m+n}^{ \pm},} \\
& {\left[I_{m}, G_{n}^{ \pm}\right]= \pm G_{m+n}^{ \pm},} \\
& {\left[G_{m}^{\varepsilon}, G_{n}^{\varepsilon^{\prime}}\right]= \begin{cases}L_{m+n}+\frac{1}{6}\left(m^{2}-\frac{1}{4}\right) \delta_{m+n, 0} c & m, n \in \frac{1}{2}+\mathbb{Z}, \\
-\varepsilon \varepsilon^{\prime}\left\{L_{m+n}+\frac{1}{6}\left(m^{2}-\frac{1}{4}\right) \delta_{m+n, 0} c\right\} & m, n \in \mathbb{Z}, \\
-\frac{1}{2} \varepsilon^{\prime}(m-n) I_{m+n}, & m \in \mathbb{Z}, n \in \frac{1}{2}+\mathbb{Z}, \\
\frac{1}{2} \varepsilon(m-n) I_{m+n}, & m \in \frac{1}{2}+\mathbb{Z}, n \in \mathbb{Z} .\end{cases} }
\end{aligned}
$$

La super-algèbre $\mathfrak{g}$ possède une décomposition triangulaire

$$
\mathfrak{g}=\mathfrak{g}_{+} \oplus \mathfrak{g}_{0} \oplus \mathfrak{g}_{-}
$$

où l'on a posé

$$
\begin{aligned}
& \mathfrak{g}_{ \pm}:=\bigoplus_{ \pm n \in \mathbb{Z}>0} \mathbb{C} L_{n} \oplus \bigoplus_{ \pm m \in-\frac{1}{2}+\mathbb{Z}_{>0}} \mathbb{C} I_{m} \oplus \bigoplus_{i=1}^{2} \bigoplus_{k_{i} \in \frac{1}{2} i+\mathbb{Z}_{\geq 0}} \mathbb{C} G_{k_{i}}^{i}, \\
& \mathfrak{g}_{0}:=\mathbb{C} L_{0} \oplus \mathbb{C} G_{0}^{2} \oplus \mathbb{C} c .
\end{aligned}
$$

\section{$2.2 \quad$ Formes Réelles}

Dans cette sous-section, on classifie les anti-involutions anti-linéaires de $\mathfrak{g}$. Ensuite, on détermine les formes réelles qui admettent des modules unitarisables non-triviaux. On se limitera des résultats à la description car les arguments donnés dans $[\mathrm{CP}]$ se généralisent à ce cas sans difficulté.

Désignons la décomposition de $\mathfrak{g}_{0}$ par rapport à la $\mathbb{Z} / 2 \mathbb{Z}$-graduation par

$$
\mathfrak{g}_{0}=\mathfrak{g}_{0}^{\overline{0}} \oplus \mathfrak{g}_{0}^{\overline{1}} .
$$

La première proposition décrit la classification des sous-algèbres de Cartan de $\mathfrak{g}:$

Proposition 2.1. La sous-algèbre $\mathfrak{g}_{0}^{\overline{0}}$ est l'unique sous-algèbre abélienne maximale de $\mathfrak{g}$ qui est ad-semi-simple et paire.

Posons

$$
S^{1}:=\{z|| z \mid=1\} \subset \mathbb{C} .
$$

Avec l'aide de Proposition 2.1, on peut montrer la proposition suivante: 
Proposition 2.2. Toute anti-involution anti-linéaire de $\mathfrak{g}$ est de l'un des types suivants:

1. Pour $\alpha \in \mathbb{R}^{\times}$et $\sigma_{i} \in\{ \pm\}(i=1,2)$,

$$
\begin{aligned}
& \theta_{\alpha ; \sigma_{1}, \sigma_{2}}^{+}\left(L_{n}\right)=\alpha^{n} L_{-n}, \quad \theta_{\alpha ; \sigma_{1}, \sigma_{2}}^{+}\left(I_{n}\right)=\sigma_{1} \sigma_{2} \alpha^{n} I_{-n}, \quad \theta_{\alpha ; \sigma_{1}, \sigma_{2}}^{+}(c)=c, \\
& \theta_{\alpha ; \sigma_{1}, \sigma_{2}}^{+}\left(G_{n}^{i}\right)=\sigma_{i} \alpha^{n} G_{-n}^{i} .
\end{aligned}
$$

2. Pour $\alpha \in S^{1}$ et $\sigma_{i} \in\{ \pm\}(i=1,2)$,

$$
\begin{aligned}
& \theta_{\alpha ; \sigma_{1}, \sigma_{2}}^{-}\left(L_{n}\right)=-\alpha^{n} L_{n}, \quad \theta_{\alpha ; \sigma_{1}, \sigma_{2}}^{-}\left(I_{n}\right)=\sigma_{1} \sigma_{2} \alpha^{n} I_{n}, \quad \theta_{\alpha ; \sigma_{1}, \sigma_{2}}^{-}(c)=-c, \\
& \theta_{\alpha ; \sigma_{1}, \sigma_{2}}^{-}\left(G_{n}^{i}\right)=\sigma_{i} \sqrt{-1} \alpha^{n} G_{n}^{i} .
\end{aligned}
$$

Grâce à la Proposition 2.1, il est naturel de considérer des $\left(\mathfrak{g}, \mathfrak{g}_{0}^{\overline{0}}\right)$-modules. La proposition suivante détermine pour quelle anti-involution anti-linéaire li existe des $\left(\mathfrak{g}, \mathfrak{g}_{0}^{\overline{0}}\right)$-modules unitarisables non-triviaux.

Proposition 2.3. Soit $V$ un $\left(\mathfrak{g}, \mathfrak{g}_{0}^{\overline{0}}\right)$-module simple non-trivial.

1. Si $V$ est unitarisable par rapport à une anti-involution anti-linéaire $\theta$, alors il existe $\alpha \in \mathbb{R}_{>0}$ tel que $\theta=\theta_{\alpha ;+,+}^{+}$.

2. Si $V$ est unitarisable par rapport à $\theta_{\alpha ;+,+}^{+}$pour un $\alpha \in \mathbb{R}_{>0}$, alors $V$ est aussi unitarisable par rapport $\grave{a} \theta=\theta_{1 ;+,+}^{+}$.

Donc, il suffit de considérer des $\left(\mathfrak{g}, \mathfrak{g}_{0}^{\overline{0}}\right)$-modules qui sont unitairisables pour $\theta_{1 ;+,+}^{+}$. En particulier, on décrit $\theta$ au lieu de $\theta_{1 ;+,+}^{+}$pour simplicité.

\subsection{Modules de type de Verma}

On présente les modules de type de Verma et on définit une forme sesqui-linéaire contravariante par rapport à $\theta$.

Posons

$$
\mathfrak{g}_{\geq}:=\mathfrak{g}_{+} \oplus \mathfrak{g}_{0} .
$$

On définit les modules de Verma et de pré-Verma comme suit: Pour $(z, h) \in$ $\mathbb{C}^{2} \cong\left(\mathfrak{g}_{0}^{\overline{0}}\right)^{*}$, soit $\mathbb{C}_{z, h}:=\mathbb{C}_{z, h}$ le $\mathfrak{g}_{0}^{\overline{0}}$-module défini par

$$
L_{0} . \mathbf{1}_{z, h}=h \mathbf{1}_{z, h}, \quad c . \mathbf{1}_{z, h}=z \mathbf{1}_{z, h}, \quad \operatorname{deg} \mathbf{1}_{z, h}=\overline{0} .
$$

On peut considérer

$$
W_{z, h}:=\operatorname{Ind}_{\mathfrak{g}_{0}^{0}}^{\mathfrak{g}_{0}^{0}} \mathbb{C}_{z, h}
$$

comme $\mathfrak{g} \geq$-module via

$$
\left.\mathfrak{g}_{+}\right|_{W_{z, h}}:=\{0\} .
$$

On pose

$$
V_{z, h}:= \begin{cases}W_{z, h} & h \neq \frac{1}{24} z \\ W_{z, h} / \mathbb{C} G_{0}^{2} \cdot \mathbf{1}_{z, h} & h=\frac{1}{24} z\end{cases}
$$


considéré aussi comme $\mathfrak{g}_{\geq}$-module. Le module de Verma $M(z, h)$ et de préVerma $N(z, h)$ sont définis par

$$
M(z, h):=\operatorname{Ind}_{\mathfrak{g}_{\geq}}^{\mathfrak{g}} V_{z, h}, \quad N(z, h):=\operatorname{Ind}_{\mathfrak{g}_{\geq}}^{\mathfrak{g}} W_{z, h} .
$$

Il est clair qu'il existe un epimorphisme $N(z, h) \rightarrow M(z, h)$.

On définit une forme contravariante sur les modules de pré-Verma comme suit: Soit

$$
\pi: U(\mathfrak{g}) \rightarrow U\left(\mathfrak{g}_{0}\right)
$$

la projection canonique par rapport à la décomposition

$$
U(\mathfrak{g})=U\left(\mathfrak{g}_{0}\right) \oplus\left\{U(\mathfrak{g}) \mathfrak{g}_{+}+\mathfrak{g}_{-} U(\mathfrak{g})\right\},
$$

et soit

$$
\pi^{\overline{0}}: U\left(\mathfrak{g}_{0}\right) \rightarrow U\left(\mathfrak{g}_{0}\right)^{\overline{0}}
$$

la projection canonique par rapport à la décomposition par la $\mathbb{Z} / 2 \mathbb{Z}$-graduation

$$
U\left(\mathfrak{g}_{0}\right)=U\left(\mathfrak{g}_{0}\right)^{\overline{0}} \oplus U\left(\mathfrak{g}_{0}\right)^{\overline{1}} .
$$

La forme contravariante $\langle\cdot, \cdot\rangle_{z, h}$ sur le module de pré-Verma $N(z, h)$ est définie par

$$
\begin{gathered}
\left\langle X .\left(1 \otimes \mathbf{1}_{z, h}\right), Y .\left(1 \otimes \mathbf{1}_{z, h}\right)\right\rangle_{z, h}:=\left(\left(\pi^{\overline{0}} \circ \pi\right)(\theta(X) Y)\right)(z, h), \\
(X, Y \in U(\mathfrak{g})) .
\end{gathered}
$$

C'est une forme hermitienne. Cette forme n'est pas toujours bien-définie. En fait, utilisant la contravariance

$$
\langle X . v, w\rangle_{z, h}=\langle v, \theta(X) . w\rangle_{z, h} \quad(X \in \mathfrak{g}, v, w \in N(z, h)),
$$

pour $X=L_{0}, c$ et $v=w=1 \otimes \mathbf{1}_{z, h}$, on obtient le lemme suivant.

Lemme 2.1. La forme $\langle\cdot, \cdot\rangle_{z, h}$ est bien-définie si et seulement si $z, h \in \mathbb{R}$.

Puisque le noyau du morphisme $N(z, h) \rightarrow M(z, h)$ est un sous-espace du radical de $\langle\cdot, \cdot\rangle_{z, h}$, cette forme induit une forme contravariante sur $M(z, h)$ qu'on désigne par le même symbole.

Dans cet article, on considèrera seulement les modules de Verma $M(z, h)$ et de pré-Verma $N(z, h)$ avec la condition $(z, h) \in \mathbb{R}^{2}$.

\subsection{Le théorème principal}

Pour $m \in \mathbb{R}_{\geq 2}, r \in \mathbb{Z}_{>0}$ et $s \in 1+2 \mathbb{Z}_{\geq 0}$, on pose

$$
z_{m}:=3\left(1-\frac{2}{m}\right), \quad h_{m: r, s}:=\frac{1}{24} z_{m}+\frac{1}{16} m\left(s-2 \frac{r}{m}\right)^{2} .
$$


Théorème 2.1. Soit $(z, h) \in \mathbb{R}^{2}$. Alors, le module de Verma $M(z, h)$ muni de la forme hermitienne $\langle\cdot, \cdot\rangle_{z, h}$ est unitarisable si et seulement si l'une des conditions ci-dessous est satifaite:

1. $z \geq 3$ et $h \geq \frac{1}{24} z$,

2. Il existe des entiers $m \in \mathbb{Z}_{>1}$ et $r \in \mathbb{Z}_{>0}$ tels que

$$
z=z_{m}, \quad h=h_{m: r, 1} \quad 0<r \leq \frac{1}{2} m .
$$

Les modules simples de plus haut poids $(z, h)$ satisfaisant la deuxième condition de ce théorème sont appelés les séries minimales unitaires.

La condition nécessaire de ce théorème a été énoncée par $\mathrm{W}$. Boucher, D. Friedan et A. Kent $[\mathrm{BFK}]$ sans démonstration. (Notons que la normalization de charge centrale dans [BFK] est different d'ici, mais les resultats-même sont equivalents. ) Le but de cet article est aussi de donner une démonstration du caractère suffisant de la condition.

\section{Réalisation des Séries Minimales Unitaires}

Dans cette section, on construit une représentation de la super-algèbre de Virasoro $N=2$ tordue sur des modules de plus haut poids simples et intégrables de l'algèbre de Lie affine $\widehat{\mathfrak{s l}}_{2}$. On en déduit que la deuxième condition de Théorème 2.1 est une condition suffisante.

\subsection{Réalisation Principale de $\widehat{\mathfrak{s l}}_{2}$ et les Operateurs de Sug- awara}

Soit $\mathfrak{a}$ l'algèbre de Lie de type $\mathfrak{s l}_{2}$ et fixons une base standard $\{e, f, h\}$ de $\mathfrak{a}$, i.e., une base qui satisfait

$$
[h, e]=2 e, \quad[h, f]=-2 f, \quad[e, f]=h .
$$

Posons $\mathfrak{a}_{0}:=\mathbb{C} h$ et $\mathfrak{a}_{1}:=\mathbb{C} e \oplus \mathbb{C} f$. Soit $(\cdot, \cdot)$ la forme bilinéaire invariante et non-dégénérée de a normalisée par $(h, h)=2$.

On désigne la réalisation principale (resp. homogène) de $\widehat{\mathfrak{s}}_{2}$ par $\widetilde{\mathfrak{a}}$ (resp. $\widehat{\mathfrak{a}}$ ), c'est-à-dire, $\widetilde{\mathfrak{a}}$ et $\widehat{\mathfrak{a}}$ sont les algèbres de Lie

$$
\begin{aligned}
& \widetilde{\mathfrak{a}}:=\mathfrak{a}_{0} \otimes \mathbb{C}\left[s^{ \pm 2}\right] \oplus \mathfrak{a}_{1} \otimes s \mathbb{C}\left[s^{ \pm 2}\right] \oplus \mathbb{C} c \oplus \mathbb{C} \rho^{\vee}, \\
& \widehat{\mathfrak{a}}:=\mathfrak{a} \otimes \mathbb{C}\left[t^{ \pm 1}\right] \oplus \mathbb{C} c \oplus \mathbb{C} d,
\end{aligned}
$$

avec les commutations

$$
\begin{aligned}
& {\left[X \otimes s^{m}, Y \otimes s^{n}\right]:=[X, Y] \otimes s^{m+n}+\frac{1}{2}(X, Y) m \delta_{m+n, 0} c,} \\
& {\left[\rho^{\vee}, X \otimes s^{m}\right]:=m X \otimes s^{m},} \\
& {[c, \widetilde{a}]:=\{0\},}
\end{aligned}
$$


et

$$
\begin{aligned}
& {\left[X \otimes t^{m}, Y \otimes t^{n}\right]:=[X, Y] \otimes t^{m+n}+(X, Y) m \delta_{m+n, 0} c} \\
& {\left[d, X \otimes t^{m}\right]:=m X \otimes s^{m}} \\
& {[c, \widehat{\mathfrak{a}}]:=\{0\}}
\end{aligned}
$$

respectivement. Un Isomorphisme entre $\widetilde{\mathfrak{a}}$ et $\widehat{\mathfrak{a}}$ est donné par

$$
\begin{aligned}
& e \otimes s^{2 m+1} \longmapsto e \otimes t^{m}, \\
& h \otimes s^{2 m} \longmapsto h \otimes t^{m}-\frac{1}{2} \delta_{m, 0} c, \\
& f \otimes s^{2 m-1} \longmapsto f \otimes t^{m}, \\
& \rho^{\vee} \longmapsto 2 d+\frac{1}{2} h \otimes 1, \quad c \longmapsto c .
\end{aligned}
$$

Soit $V$ un $\widehat{\mathfrak{s l}}_{2}$-module de plus haut poids de charge centrale $p \in \mathbb{Z}_{>0}$ et considérons les séries génératrices d'opérateurs sur $V$ :

$$
\begin{aligned}
& J^{0}(z):=\frac{1}{2} \sum_{n \equiv 1}(e+f) \otimes s^{n} z^{-n-1} \\
& J^{ \pm}(z):=\frac{1}{2} \sum_{n \equiv 0} h \otimes s^{n} z^{-n-1} \pm \frac{1}{2} \sum_{n \equiv 1}(-e+f) \otimes s^{n} z^{-n-1}
\end{aligned}
$$

et

$$
X(z):=\sum_{n \in \mathbb{Z}} e \otimes t^{n} z^{-n-1}
$$

pour $X \in\{e, f, h\}$. Ici, $n \equiv i$ signifie la condition $n \in \mathbb{Z}$ telle que $n \equiv i \bmod 2$. Pour une série d'opérateurs $f(z)=\sum_{n \in \mathbb{Z}} f_{n} z^{n}$ et un sous-ensemble $I \subset \mathbb{R}$, on pose

$$
f(z)_{I}:=\sum_{n \in I \cap \mathbb{Z}} f_{n} z^{n}
$$

En particulier, pour $I_{+}:=\mathbb{R}_{\geq 0}$ et $I_{-}:=\mathbb{R}_{<0}$, on les désigne par $f(z)_{+}$et $f(z)_{-}$, respectivement. Pour deux séries d'opérateurs $X(z)$ et $Y(z)$, on définit le produit ordonné par

$$
{ }_{\circ}^{\circ} X(z) Y(z)_{\circ}^{\circ}:=X(z)_{+} Y(z)+Y(z) X(z)_{-} .
$$

Remarquons que pour chaqune des deux séries présentées ci-dessus, le produit ordonné est bien-défini. 
Rappelons que les opérateurs de Sugawara sont définis par

$$
\begin{aligned}
T^{p r}(z) & :=\frac{1}{2(p+2)}\left\{{ }_{\circ}^{\circ} J^{+}(z) J^{-}(z)_{\circ}^{\circ}+{ }_{\circ}^{\circ} J^{-}(z) J^{+}(z)_{\circ}^{\circ}+2_{\circ}^{\circ} J^{0}(z)_{\circ}^{2 \circ}+\frac{1}{16} p z^{-2}\right\} \\
& :=\sum_{m \in \mathbb{Z}}\left(L_{m}^{p r}-\frac{1}{32} c_{p} \delta_{m, 0} \mathrm{id}\right) z^{-2 m-2}, \\
T^{h o m}(z) & :=\frac{1}{2(p+2)}\left\{{ }_{\circ}^{\circ} e(z) f(z)_{\circ}^{\circ}+{ }_{\circ}^{\circ} f(z) e(z)_{\circ}^{\circ}+\frac{1}{2} \circ h(z)_{\circ}^{2 \circ}\right\} \\
& :=\sum_{m \in \mathbb{Z}} L_{m}^{h o m} z^{-m-2},
\end{aligned}
$$

où le nombre $c_{p}$ est donné par $c_{p}:=\frac{3 p}{p+2}$. Par définition, on peut facilement voir que la relation entre ces deux opérateurs de Sugawara est donnée par

$$
\sum_{m \in \mathbb{Z}} L_{m}^{p r} z^{-2 m-2}=z^{2} T^{h o m}\left(z^{2}\right)-\frac{1}{4} z^{-1} h\left(z^{2}\right)+\frac{1}{16} p z^{-2} .
$$

\subsection{Caractères Spécialisés des $\widehat{\mathfrak{s l}}_{2}$-modules intégrables}

Selon une idée de V. Rittenberg et A. Schwimmer [RS], on calcule la spécialisation principale de caractères des $\widehat{\mathfrak{s l}}_{2}$-modules intégrables et on les compare aux caractères des séries minimales unitaires.

Fixons un entier positif $p \in \mathbb{Z}_{>0}$. Pour chaque $I \in \mathbb{Z}_{\geq 0}$ tel que $I \leq p$, soit $\Lambda_{p, I}:=(p-1) \Lambda_{0}+I \Lambda_{1} \in \mathfrak{h}^{*}$ un poids dominant et entier où $\Lambda_{i}(i=0,1)$ sont les poids fondamentaux de $\widehat{\mathfrak{s l}}_{2}$. Ici, $\mathfrak{h} \subset \widehat{\mathfrak{s l}}_{2}$ est la sous-algèbre de Cartan. Soit $L\left(\Lambda_{p, I}\right)$ le $\widehat{\mathfrak{s l}}_{2}$-module simple de plus haut poids $\Lambda_{p, I}$ et posons

$$
\chi_{p, I}(\tau):=\operatorname{tr}_{L\left(\Lambda_{p, I}\right)}\left(q^{L_{0}^{p r}-\frac{1}{24} c_{p}}\right),
$$

où $\tau$ et $q$ sont liés par la relation $q=e^{2 \pi \sqrt{-1} \tau}(\operatorname{Im} \tau>0)$.

D'après la formule du caractère de Weyl et Kac $[\mathrm{K}]$, on obtient la formule

$$
\operatorname{ch} L\left(\Lambda_{p, I}\right)=e^{\Lambda_{p, I}} \frac{\sum_{n \in \mathbb{Z}} e^{m n \alpha_{1}-\left(r n+m n^{2}\right) \delta}-\sum_{n \in \mathbb{Z}} e^{(m-r) n \alpha_{1}-\left(-r n+m n^{2}\right) \delta}}{\prod_{n>0}\left(1-e^{-\alpha_{1}+(n-1) \delta}\right)\left(1-e^{-\alpha_{1}+n \delta}\right)\left(1-e^{-n \delta}\right)},
$$

où l'on a posé

$$
m:=p+2, \quad r:=I+1 .
$$

Soit $\left|\Lambda_{p, I}\right\rangle \in L\left(\Lambda_{p, I}\right)$ des vecteurs de plus haut poids non nuls. Par (3), on obtient

$$
L_{0}^{p r} \cdot\left|\Lambda_{p, I}\right\rangle=\left[\frac{(m-2 r)^{2}}{16 m}+\frac{1}{24} \cdot 3\left(1-\frac{2}{m}\right)\right]\left|\Lambda_{p, I}\right\rangle .
$$

Donc, par la spécialisation

$$
e^{\Lambda_{p, I}} \longmapsto q^{h_{m: r, 1}}, \quad e^{-\alpha_{1}} \longmapsto q^{\frac{1}{2}}, \quad e^{-\delta} \longmapsto q,
$$


on obtien la formule suivante

$$
\chi_{p, I}(\tau)=\frac{\sum_{n \in \mathbb{Z}} q^{m\left[n+\frac{1}{4 m}(2 r-m)\right]^{2}}-\sum_{n \in \mathbb{Z}} q^{m\left[n+\frac{1}{4 m}(2 r+m)\right]^{2}}}{\prod_{n>0}\left(1-q^{n}\right)\left(1-q^{n-\frac{1}{2}}\right)^{2}} .
$$

D'après K. Iohara et Y. Koga $[\mathrm{IK}]$, pour le cas $I \neq \frac{1}{2} p, 2 \chi_{p, I}(\tau)$ coïncide avec le caractère normalisé de $L\left(z_{m}, h_{m: r, 1}\right)$, et pour le cas $I=\frac{1}{2} p, \chi_{p, I}(\tau)$ coïncide avec le caractère normalisé de $L\left(z_{m}, h_{m: \frac{1}{2} m, 1}\right)$. De plus, on a la symétrie $\chi_{p, p-I}(\tau)=\chi_{p, I}(\tau)$.

Dans les sous-sections ci-dessous, on définit une structure de $\mathfrak{g}$-module sur les $\widehat{\mathfrak{s l}}_{2}$-modules

$$
V_{p, I}:=\left\{\begin{array}{cl}
L\left(\Lambda_{p, I}\right) \oplus L\left(\Lambda_{p, p-I}\right) & I \neq \frac{1}{2} p \\
L\left(\Lambda_{p, \frac{1}{2} p}\right) & I=\frac{1}{2} p .
\end{array}\right.
$$

\subsection{Opération de la Super-Algèbre de Virasoro $N=2$ Tor- due}

En utilisant l'algèbre $Z$ de J. Lepowsky et R. L. Wilson [LW], on présente une structure de $\mathfrak{g}$-module sur $V_{p, I}$. C'est une version tordue de [ZF]. Tous les opérateurs ici sont des opérateurs sur $V_{p, I}$. On suppose $p \in \mathbb{Z}_{>0}$.

Par simplicité, notons

$$
a(z)=\sum_{n \equiv 1} a_{n} z^{-n-1}:=\frac{2}{p^{\frac{1}{2}}} J^{0}(z), \quad \varphi(z):=-\sum_{n \equiv 1} \frac{a_{n}}{n} z^{-n} .
$$

Alors, les coefficients de la série d'opérateurs

$$
T_{0}(z)=\sum_{m \in \mathbb{Z}}\left(L_{m}^{0}-\frac{1}{32} \delta_{m, 0} \mathrm{id}\right) z^{-2 m-2}:=\frac{1}{4}{ }_{\circ}^{\circ} a(z)_{\circ}^{2 \circ}+\frac{1}{32} z^{-2},
$$

engendrent l'algèbre de Virasoro de charge centrale 1. Pour $\lambda \in \mathbb{C}$, posons

$$
V_{\lambda}(z):=(2 z)^{-\frac{1}{4} \lambda^{2}} \exp \left(\lambda \varphi(z)_{+}\right) \exp \left(\lambda \varphi(z)_{-}\right) .
$$

On peut montrer que le champ $V_{\lambda}(z)$ est un champ conforme de dimension $\frac{1}{4} \lambda^{2}$, c'est-à-dire, qu'il satisfait la relation

$$
\left[L_{m}^{0}, V_{\lambda}(z)\right]=\frac{1}{2} z^{2 m+1}\left\{\partial_{z}+\frac{1}{4} \lambda^{2}(2 m+1) z^{-1}\right\} V_{\lambda}(z)
$$

Suivant $[\mathrm{LW}]$, on pose

$$
\psi_{ \pm}(z):=2 p^{-\frac{1}{2}}(2 z)^{\frac{1}{p}} \exp \left(\mp \frac{2}{p^{\frac{1}{2}}} \varphi(z)_{+}\right) J^{ \pm}(z) \exp \left( \pm \frac{2}{p^{\frac{1}{2}}} \varphi(z)_{-}\right) .
$$


Alors, $\psi_{ \pm}(z)$ commutent avec $a(w)$. De plus, on peut montrer que $\psi_{+}(z)$ et $\psi_{-}(w)$ satisfont le développement en produits d'opérateurs

$$
\psi_{+}(z) \psi_{-}(w) \sim(z-w)^{-\frac{2(p-1)}{p}}\left[1+8 \frac{\Delta_{1}}{\widetilde{c}_{p}} T_{p}(w)(z-w)^{2}+O(z-w)^{3}\right],
$$

et la relation

$$
\left[L_{m}^{p}, \psi_{ \pm}(w)\right]=\frac{1}{2} w^{2 m+1}\left\{\partial_{w}+\Delta_{1}(2 m+1) w^{-1}\right\} \psi_{ \pm}(w) .
$$

Ici, $\Delta_{1}:=\frac{p-1}{p}$ est la dimension conforme de $\psi_{ \pm}(w)$,

$$
\widetilde{c}_{p}:=c_{p}-1=2\left(\frac{p-1}{p+2}\right)
$$

est la charge centrale de l'algèbre de Virasoro

$$
T_{p}(z)=\sum_{m \in \mathbb{Z}}\left(L_{m}^{p}-\frac{1}{32} \widetilde{c}_{p} \delta_{m, 0}\right) z^{-2 m-2}:=T^{p r}(z)-T_{0}(z) .
$$

L'automorphisme de Dynkin de $\widehat{\mathfrak{s l}}_{2}$ induit un automorphisme $\nu \in \operatorname{End}_{\mathbb{C}}\left(V_{p, I}\right)$. Il est clair que $\nu$ satisfait

$$
\nu \circ J^{0}(z)=J^{0}(z) \circ \nu, \quad \nu \circ J^{ \pm}(z)=-J^{ \pm}(z) \circ \nu .
$$

Sans perte de généralité, on supposera que les vecteurs $\left|\Lambda_{p, I}\right\rangle$ et $\left|\Lambda_{p, p-I}\right\rangle$ satisfont

$$
\nu\left(\left|\Lambda_{p, I}\right\rangle\right)=\left|\Lambda_{p, p-I}\right\rangle, \quad \nu\left(\left|\Lambda_{p, p-I}\right\rangle\right)=\left|\Lambda_{p, I}\right\rangle
$$

pour tout $0 \leq I \leq p$. (Le cas $I=\frac{1}{2} p$ est aussi valable par définition de $L\left(\Lambda_{p, \frac{1}{2} p}\right)$ comme le quotient simple d'un module de Verma.)

On présente les séries de générateurs de $\mathfrak{g}$ comme suit:

$$
\begin{aligned}
T(z) & :=\sum_{m \in \mathbb{Z}}\left(L_{m}-\frac{1}{32} c \delta_{m, 0}\right) z^{-2 m-2}, \\
I(z) & :=\sum_{m \in \frac{1}{2}+\mathbb{Z}} I_{m} z^{-2 m-1}, \\
G^{ \pm}(z) & :=\sum_{m \in \frac{1}{2} \mathbb{Z}} G_{m}^{ \pm} z^{-2 m-\frac{3}{2}} .
\end{aligned}
$$

Posons

$$
\beta_{p}:=\left(\frac{6}{c_{p}}\right)^{\frac{1}{2}}, \quad \mu_{p}:=\sqrt{-1}\left(\frac{c_{p}}{12}\right)^{\frac{1}{2}}
$$


Théorème 3.1. 1. La super-algèbre de Lie $\mathfrak{g}$ opère sur $V_{p, I}$ via

$$
\begin{aligned}
& T(z) \longmapsto T^{p r}(z), \\
& I(z) \longmapsto \beta_{p}^{-1} a(z), \\
& G^{ \pm}(z) \longmapsto \mu_{p} \psi_{ \pm}(z) V_{ \pm \beta_{p}}(z) \nu, \\
& c \longmapsto c_{p} \operatorname{id}_{V_{p, I}} .
\end{aligned}
$$

2. Pour cette opération, $V_{p, I}$ est isomorphe au $\mathfrak{g}$-module simple de plus haut poids $\left(z_{m}, h_{m: r, 1}\right)$.

Notons que le deuxième énoncé est un corollaire de la sous-section précédente.

\subsection{Unitarisabilité du g-module $V_{p, I}$}

Soit $\omega$ l'anti-involution anti-linéaire de $\mathfrak{a}$ définie par

$$
\omega(e):=f, \quad \omega(f):=e, \quad \omega(h):=h .
$$

$\omega$ se prolonge à l'anti-involution anti-linéaire $\widetilde{\omega}$ de $\widetilde{\mathfrak{a}}$ par

$$
\widetilde{\omega}\left(X \otimes s^{n}\right):=\omega(X) \otimes s^{-n}, \quad \widetilde{\omega}(c):=c, \quad \widetilde{\omega}\left(\rho^{\vee}\right):=\rho^{\vee} .
$$

Soit $(\cdot, \cdot)$ la forme hermitienne contravariante par rapport à $\widetilde{\omega}$ satisfaisant

$$
\begin{aligned}
& (\lambda u, v)=\bar{\lambda}(u, v) \quad \lambda \in \mathbb{C}, u, v \in V_{p, I}, \\
& \left(\left|\Lambda_{p, I}\right\rangle,\left|\Lambda_{p, I}\right\rangle\right)=\left(\left|\Lambda_{p, p-I}\right\rangle,\left|\Lambda_{p, p-I}\right\rangle=1,\right. \\
& \left(\left|\Lambda_{p, I}\right\rangle,\left|\Lambda_{p, p-I}\right\rangle\right)=0 \quad \text { si } I \neq \frac{1}{2} p, \\
& (x . u, v)=(u, \widetilde{\omega}(x) \cdot v) \quad x \in \widetilde{\mathfrak{a}}, u, v \in V .
\end{aligned}
$$

On sait que $V_{p, I}$ muni de la forme $(\cdot, \cdot)$ est un $\widehat{\mathfrak{s l}}_{2}$-module unitaire.

Pour $X \in \operatorname{End}_{\mathbb{C}}\left(V_{p, I}\right)$, désignons par $X^{\dagger}$ l'operateur conjugé hermitien de $X$ par rapport à $(\cdot, \cdot)$. En particulier, on a

$$
X^{\dagger}=\widetilde{\omega}(X) \quad(X \in \widetilde{\mathfrak{a}}), \quad \nu^{\dagger}=\nu .
$$

D'après la réalisation donnée par le Théorème 3.1 , on peut montrer les formules suivantes:

$$
\left(L_{m}\right)^{\dagger}=L_{-m}, \quad\left(I_{m}\right)^{\dagger}=I_{-m}, \quad\left(G_{m}^{i}\right)^{\dagger}=G_{-m}^{i}, \quad(c)^{\dagger}=c .
$$

C'est-à-dire, la conjugaison hermitienne coïncide avec l'anti-involution antilinéaire $\theta$ sur $\mathfrak{g} \subset \operatorname{End}_{\mathbb{C}}\left(V_{p, I}\right)$.

Proposition 3.1 (cf. $[\mathrm{K}]$ ). Le $\mathfrak{g}$-module $V_{p, I}$ est unitaire. 
Soit $(z, h)=\left(z_{m}, h_{m: r, 1}\right)$ un poids de $\mathfrak{g}$ qui satisfait la deuxième condition de Théorème 2.1. Si $m>2$, la Proposition 3.1 indique que le module $M(z, h)$ est unitarisable. Si $m=2$, on a $(z, h)=(0,0)$ et dans ce cas, le quotient simple de $M(z, h)$ est un module trivial de dimension 1 qui est clairement unitaire. Par consequent, on obtient le corollaire suivant du Théorème 3.1 et de la Proposition 3.1:

Corollaire 3.1. Soit $(z, h) \in \mathbb{R}^{2}$ un poids de $\mathfrak{g}$ qui satisfait la deuxième condition de Théorème 2.1. Alors, le module $M(z, h)$ muni de la forme $\langle\cdot, \cdot\rangle_{z, h}$ est unitarisable.

\section{Formule du Déterminant}

Dans cette section, on décrit les formules du déterminant de la forme hermitienne sur un module de Verma et sur son quotient. On utilisera ces formules pour démontrer le Théorème 2.1 .

\subsection{Module de Verma}

Ici, on rappelle la formule du déterminant de $\langle\cdot, \cdot\rangle_{z, h}$ et une partie de la démonstration qui sera utilisée dans la section suivante.

Le module de Verma $M(z, h)$ admet la décomposition en sous-espaces de poids

$$
M(z, h)=\bigoplus_{n \in \frac{1}{2} \mathbb{Z}_{\geq 0}} M(z, h)_{n}, \quad M(z, h)_{n}:=\left\{u \mid L_{0} \cdot u=(h+n) u\right\} .
$$

Cette décomposition est compatible avec celle de la $\mathbb{Z} / 2 \mathbb{Z}$-graduation

$$
M(z, h)^{\tau}=\bigoplus_{n \in \frac{1}{2} \mathbb{Z}_{\geq 0}} M(z, h)_{n}^{\tau}, \quad M(z, h)_{n}^{\tau}:=M(z, h)_{n} \cap M(z, h)^{\tau},
$$

où $M(z, h)^{\tau}$ est le sous-espace de $M(z, h)$ de parité $\tau \in \mathbb{Z} / 2 \mathbb{Z}$. Par définition et par la contravariance de la forme $\langle\cdot, \cdot\rangle_{z, h}$, on a

$$
\left.\langle\cdot, \cdot\rangle\right|_{M(z, h)_{m}^{\sigma} \times M(z, h)_{n}^{\tau}}=0 \quad \text { si }(\sigma, m) \neq(\tau, n) .
$$

Donc, il suffit de considérer la restriction

$$
\langle\cdot, \cdot\rangle_{z, h ; n}^{\tau}:=\left.\langle\cdot, \cdot\rangle_{z, h}\right|_{M(z, h)_{n}^{\tau} \times M(z, h)_{n}^{\tau}}, \quad\left(\tau \in \mathbb{Z} / 2 \mathbb{Z}, n \in \frac{1}{2} \mathbb{Z}_{\geq 0}\right) .
$$

Puisque chaque sous-espace gradué de $M(z, h)$ est de dimension finie d'après le théorème de Poincaré-Birkhoff-Witt, on peut considérer le discriminant de la forme $\langle\cdot, \cdot\rangle_{z, h ; n}^{\tau}$, que nous notons par $\operatorname{det}(z, h)_{n}^{\tau}$.

Soient $\left\{P_{\mathrm{T}}(n)\right\}_{n \in \frac{1}{2} \mathbb{Z}_{\geq 0}} \subset \mathbb{Z}_{\geq 0}$ les nombres entiers défini par

$$
\sum_{n \in \frac{1}{2} \mathbb{Z}_{\geq 0}} P_{\mathrm{T}}(n) x^{n}=\prod_{k=1}^{\infty} \frac{\left(1+x^{\frac{1}{2} k}\right)}{\left(1-x^{\frac{1}{2} k}\right)} \in \mathbb{Z}\left[\left[x^{\frac{1}{2}}\right]\right] .
$$


Remarque 4.1. Par définition, $P_{\mathrm{T}}(0)=1$ et $P_{\mathrm{T}}(n) \in 2 \mathbb{Z}_{>0}$ pour tout $n \in$ $\frac{1}{2} \mathbb{Z}_{>0}$.

Rappelons la formule du determinant $\operatorname{det}(z, h)_{n}^{\tau}$ démontrée dans [IK]:

Théorème 4.1. Supposons que $n \in \frac{1}{2} \mathbb{Z}_{>0}$ et $\tau \in \mathbb{Z} / 2 \mathbb{Z}$.

1. Si $h \neq \frac{1}{24} z$, on $a$

$$
\operatorname{det}(z, h)_{n}^{\tau}=C_{n}\left(h-\frac{1}{24} z\right)^{\frac{1}{2} P_{\mathrm{T}}(n)} \prod_{\substack{r \in \mathbb{Z}_{>0}, s \in-1+2 \mathbb{Z}_{>0} \\ 1 \leq r s \leq 2 n}}\left\{f_{r, s}(z, h)\right\}^{P_{\mathrm{T}}\left(n-\frac{1}{2} r s\right)},
$$

où l'on a posé

$$
f_{r, s}(z, h):=2\left(\frac{1}{3} z-1\right)\left(h-\frac{1}{24} z\right)+\frac{1}{4}\left[r\left(\frac{1}{3} z-1\right)+s\right]^{2},
$$

et $\left\{C_{n}\right\} \subset \mathbb{R}_{>0}$ est un sous-ensemble dépendant d'un choix de base.

2. Si $h=\frac{1}{24} z$, on $a$

$$
\operatorname{det}\left(z, \frac{1}{24} z\right)_{n}^{\tau}=C_{n}^{\prime} \prod_{\substack{r \in \mathbb{Z}_{>0}, s \in-1+2 \mathbb{Z}_{>0} \\ 1 \leq r s \leq 2 n}}\left\{F_{r, s}(z)\right\}^{P_{\mathrm{T}}\left(n-\frac{1}{2} r s\right)},
$$

où l'on a posé

$$
F_{r, s}(z):=\frac{1}{2}\left[r\left(\frac{1}{3} z-1\right)+s\right],
$$

et $\left\{C_{n}^{\prime}\right\} \subset \mathbb{R}_{>0}$ est un sous-ensemble dépendant d'un choix de base.

Remarquons qu'on a la relation

$$
f_{r, s}\left(z, \frac{1}{24} z\right)=F_{r, s}(z)^{2} .
$$

En fait, pour démontrer le Théorème 4.1, on doit montrer un peu plus que Lemme 20 de [IK]. C'est ce que nous précisons ci-dessous.

Posons

$$
\mathcal{I}:=\left\{(i, \epsilon) \mid i \in \frac{1}{2} \mathbb{Z}_{>0}, \epsilon \in \mathbb{Z} / 2 \mathbb{Z}\right\},
$$

et définissons l'ordre total $\prec$ sur $\mathcal{I}$ par

$$
\left(\frac{1}{2}, \overline{0}\right) \prec\left(\frac{1}{2}, \overline{1}\right) \prec(1, \overline{0}) \prec(1, \overline{1}) \prec\left(\frac{3}{2}, \overline{0}\right) \prec \cdots .
$$

Ensuite, pour $n \in \frac{1}{2} \mathbb{Z}_{>0}$ et $\tau \in \mathbb{Z} / 2 \mathbb{Z}$, posons

$$
\mathcal{P}_{n}^{\tau}:=\left\{\begin{array}{l|l}
\left(\left(i_{k}, \epsilon_{k}\right), \cdots,\left(i_{1}, \epsilon_{1}\right)\right) & \begin{array}{l}
k \in \mathbb{Z}_{>0},\left(i_{j}, \epsilon_{j}\right) \in \mathcal{I}, \\
\sum_{j=1}^{k} i_{j}=n, \sum_{j=1}^{k} \epsilon_{j}=\tau, \\
\left(i_{j}, \epsilon_{j}\right)\left(i_{j+1}, \epsilon_{j+1}\right), \\
\left(i_{j}, \epsilon_{j}\right) \neq\left(i_{j+1}, \epsilon_{j+1}\right) \text { if } \epsilon_{j}=\overline{1}
\end{array}
\end{array}\right\} .
$$


Alors, l'ensemble $\mathcal{P}_{n}^{\tau}$ paramètere une base de Poincaré-Birkhoff-Witt de

$$
U\left(\mathfrak{g}_{-}\right)_{-n}^{\tau}:=\left\{x \in U\left(\mathfrak{g}_{-}\right) \mid \operatorname{deg} x=\tau,\left[L_{0}, x\right]=n x\right\} .
$$

En fait, si l'on pose

$$
x_{(i, \epsilon)}:= \begin{cases}L_{-i} & \text { si } i \in \mathbb{Z} \text { et } \epsilon=\overline{0}, \\ I_{-i} & \text { si } i \in \frac{1}{2}+\mathbb{Z} \text { et } \epsilon=\overline{0} \\ G_{-i}^{1} & \text { si } i \in \frac{1}{2}+\mathbb{Z} \text { et } \epsilon=\overline{1} \\ G_{-i}^{2} & \text { si } i \in \mathbb{Z} \text { et } \epsilon=\overline{1}\end{cases}
$$

pour $(i, \epsilon) \in \mathcal{I}$, l'on a

$$
U\left(\mathfrak{g}_{-}\right)_{-n}^{\tau}=\bigoplus_{\left(\left(i_{k}, \epsilon_{k}\right), \cdots,\left(i_{1}, \epsilon_{1}\right)\right) \in \mathcal{P}_{n}^{\tau}} \mathbb{C} x_{\left(i_{k}, \epsilon_{k}\right)} \cdots x_{\left(i_{1}, \epsilon_{1}\right)} .
$$

Donc, on obtinet une base du module de Verma comme suit:

1. $h \neq \frac{1}{24}$. Pour $\mathbb{I}=\left(\left(i_{k}, \epsilon_{k}\right), \cdots,\left(i_{1}, \epsilon_{1}\right)\right) \in \mathcal{P}_{n}^{\tau}$ and $\delta \in\{0,1\}$, posons

$$
m_{\mathbb{I}}^{\delta}:=x_{\left(i_{k}, \epsilon_{k}\right)} \cdots x_{\left(i_{1}, \epsilon_{1}\right)}\left(G_{0}^{2}\right)^{\delta} \cdot\left(1 \otimes \mathbf{1}_{z, h}^{\overline{0}}\right) .
$$

Alors,

$$
\mathcal{B}_{n}^{\tau}:=\left\{m_{\mathbb{I}}^{\delta} \mid \delta \in\{0,1\}, \mathbb{I} \in \mathcal{P}_{n}^{\tau-\delta \overline{1}}\right\}
$$

forme une base de $M(z, h)_{n}^{\tau}$.

2. $h=\frac{1}{24}$. Pour $\mathbb{I}=\left(\left(i_{k}, \epsilon_{k}\right), \cdots,\left(i_{1}, \epsilon_{1}\right)\right) \in \mathcal{P}_{n}^{\tau}$, posons

$$
m_{\mathbb{I}}:=x_{\left(i_{k}, \epsilon_{k}\right)} \cdots x_{\left(i_{1}, \epsilon_{1}\right)} \cdot\left(1 \otimes \mathbf{1}_{z, h}^{\overline{0}}\right) .
$$

Alors,

$$
\mathcal{B}_{n}^{\tau}:=\left\{m_{\mathbb{I}} \mid \mathbb{I} \in \mathcal{P}_{n}^{\tau}\right\}
$$

forme une base de $M(z, h)_{n}^{\tau}$.

Pour $\mathbb{I}=\left(\left(i_{k}, \epsilon_{k}\right), \cdots,\left(i_{1}, \epsilon_{1}\right)\right) \in \mathcal{P}_{n}^{\tau}$, on pose

$$
|\mathbb{I}|:=k-\sharp\left\{j \mid i_{j} \in \frac{1}{2}+\mathbb{Z} \text { et } \epsilon_{j}=\overline{0}\right\} .
$$

Lemme 4.1. 1. Supposons que $h \neq \frac{1}{24} z$. Pour $m_{\mathbb{I}}^{\delta}, m_{\mathbb{J}}^{\delta^{\prime}} \in \mathcal{B}_{n}^{\tau}$, on a

(a) $\left\langle m_{\mathbb{I}}^{\delta}, m_{\mathbb{I}}^{\delta}\right\rangle_{z, h}=h^{|\mathbb{I}|+\delta}\left(c_{\mathbb{I}}^{\delta}+o(1)\right)$ avec une constante $c_{\mathbb{I}}^{\delta} \in \mathbb{R}_{>0}$.

(b) $\left\langle m_{\mathbb{I}}^{\delta}, m_{\mathbb{J}}^{\delta^{\prime}}\right\rangle_{z, h}=o\left(h^{\frac{1}{2}\left(|\mathbb{I}|+|\mathbb{J}|+\delta+\delta^{\prime}\right)}\right)$, si $m_{\mathbb{I}}^{\delta} \neq m_{\mathbb{J}}^{\delta^{\prime}}$.

2. Supposons que $h=\frac{1}{24} z$. Alors, pour $m_{\mathbb{I}}, m_{\mathbb{J}} \in \mathcal{B}_{n}^{\tau}$, on a

(a) $\left\langle m_{\mathbb{I}}, m_{\mathbb{I}}\right\rangle_{z, h}=h^{|\mathbb{I}|}\left(c_{\mathbb{I}}+o(1)\right)$ avec une constante $c_{\mathbb{I}} \in \mathbb{R}_{>0}$.

(b) $\left\langle m_{\mathbb{I}}, m_{\mathbb{J}}\right\rangle_{z, h}=o\left(h^{\frac{1}{2}(|\mathbb{I}|+|\mathbb{J}|)}\right)$, si $m_{\mathbb{I}} \neq m_{\mathbb{J}}$.

Ici, o est un symbole de Landau par rapport à $h \longrightarrow \infty$. 


\subsection{Quotient du Module de Verma}

Ici, on considère le cas où le poids $(z, h)$ peut être écrit comme $(z, h)=\left(z_{m}, h_{m: r_{0}, s_{0}}\right)$ pour un nombre réel positif $m \in \mathbb{R}_{>0}$ et des nombres entiers $\left(r_{0}, s_{0}\right) \in \mathbb{Z}_{>0} \times$ $\left(1+2 \mathbb{Z}_{\geq 0}\right)$. On suppose que $h \neq \frac{1}{24} z$ et on pose $n_{0}:=\frac{1}{2} r_{0} s_{0}$. Excepté quelques cas finis, on sait qu'un morphisme $M\left(z, h+n_{0}\right) \longrightarrow M(z, h)$ est injectif d'après le Théorème 47 de $[\mathrm{IK}]$. On désigne ce conoyau par $\bar{M}(z, h)$. La projection canonique $M(z, h) \rightarrow \bar{M}(z, h)$ induit la decomposition

$$
\bar{M}(z, h)=\bigoplus_{(n, \tau) \in \frac{1}{2} \mathbb{Z} \geq 0 \times \mathbb{Z} / 2 \mathbb{Z}} \bar{M}(z, h)_{n}^{\tau},
$$

où $\bar{M}(z, h)_{n}^{\tau}$ est l'image de $M(z, h)_{n}^{\tau}$. Cette projection induit aussi une forme hermitienne dont le discriminant est noté $\overline{\operatorname{det}}(z, h)_{n}^{\tau}$.

Théorème 4.2. Supposons que $h \neq \frac{1}{24} z$. Pour $n \in \frac{1}{2} \mathbb{Z}_{>0}$ tel que $n \geq n_{0}$ et $\tau \in \mathbb{Z}_{2}$, on $a$

$$
\begin{aligned}
& \overline{\operatorname{det}}(z, h)_{n}^{\tau} \times \operatorname{det}\left(z, h+n_{0}\right)_{n-n_{0}}^{\tau} \\
= & \bar{C}_{n}\left(h-\frac{1}{24} z\right)^{\frac{1}{2} P_{T}(n)} \prod_{\substack{r \in \mathbb{Z}_{>0}, s \in 1+2 \mathbb{Z}_{\geq 0} \\
1 \leq r s \leq 2 n \\
(r, s) \neq\left(r_{0}, s_{0}\right)}}\left\{f_{r, s}(z, h)\right\}^{P_{T}\left(n-\frac{1}{2} r s\right)} .
\end{aligned}
$$

où $\left\{\bar{C}_{n}\right\} \subset \mathbb{R}_{>0}$ est un sous-ensemble dépendant d'un choix de base.

\section{Démonstration}

Dans cette section, en analysant la formule du déterminant de la forme hermitienne, on démontre le Théorème 2.1. On pose $|\mathrm{vac}\rangle:=1 \otimes \mathbf{1}_{z^{\prime}, h^{\prime}}^{\overline{0}}$.

\subsection{Cas général et la première condition}

Le premier lemme est

Lemme 5.1. Si la forme $\langle\cdot, \cdot\rangle_{z, h}$ est positive semi-définie, alors on a

$$
h \geq \frac{1}{24} z \geq 0 .
$$

Démonstration. Pour $n \in \mathbb{Z}_{>0}$, on a

$$
\left.\left\langle L_{-n} \mid \operatorname{vac}\right\rangle, L_{-n}|\operatorname{vac}\rangle\right\rangle_{z, h}=2 n\left(h+\frac{1}{24}\left(n^{2}-1\right) z\right) \geq 0 .
$$

Considérons le cas $n$ suffisament grand, on obtient $z \geq 0$. De plus, on a

$$
\left.\left\langle G_{0}^{2} \mid \mathrm{vac}\right\rangle, G_{0}^{2}|\mathrm{vac}\rangle\right\rangle_{z, h}=h-\frac{1}{24} z \geq 0 \text {. }
$$


Remarquons que $f_{r, s}(z, h) \geq 0$ pour tous $z \geq 3$ et $h \geq \frac{1}{24} z$.

Lemme 5.2. Supposons que $z \geq 3$ et $h \geq \frac{1}{24} z$. Alors, la forme $\langle\cdot, \cdot\rangle_{z, h}$ est positive semi-définie.

Démonstration. En fait, Lemme 4.1 implique que $\langle\cdot, \cdot\rangle_{z, h}$ est positive pour $z>3$ fixé et $h$ suffisament grand et la signature de cette forme ne change pas dans le domaine de ce lemme.

Donc, dans le cas de $z \geq 3$, on a montré que la première condition du Théorème 2.1 est nécessaire et suffisante.

Dorénavant, on suppose que le poids $(z, h)$ satisfait

$$
0 \leq z<3, \quad h-\frac{1}{24} z \geq 0 .
$$

En général, la forme $\langle\cdot, \cdot\rangle_{z, h}$ n'est pas positive semi-définie s'il existe $n \in$ $\frac{1}{2} \mathbb{Z}_{>0}$ tel que $\operatorname{det}(z, h)_{n}^{\tau}<0$. Nous excluerons de tels cas par l'argument comme suit:

Pour $(z, h)$ fixé, on cherche $(r, s) \in \mathbb{Z}_{>0} \times\left(1+2 \mathbb{Z}_{\geq 0}\right)$ telle que $f_{r, s}(z, h)<0$ (ou $\left.F_{r, s}(z, h)<0\right)$ et que $f_{r^{\prime}, s^{\prime}}(z, h)>0\left(\right.$ ou $\left.F_{r^{\prime}, s^{\prime}}(z, h)>0\right)$ pour toute $\left(r^{\prime}, s^{\prime}\right) \in$ $\mathbb{Z}_{>0} \times\left(1+2 \mathbb{Z}_{\geq 0}\right)$ satisfaisant $r^{\prime} s^{\prime} \leq r s$ et $\left(r^{\prime}, s^{\prime}\right) \neq(r, s)$. Si une telle $(r, s)$ existe, on a $\operatorname{det}(z, h)_{\frac{1}{2} r s}^{\tau}<0$ par le Théorème 4.1 et Remarque 4.1 .

\subsection{Le cas $h=\frac{1}{24} z$}

D'après l'hypothèse, il existe $m \in \mathbb{R}_{\geq 2}$ tel que $z=z_{m}$, où $z_{m}$ est défini dans (1). Alors, on a

$$
F_{r, s}\left(z_{m}, \frac{1}{24} z_{m}\right)=\frac{1}{2}\left(-\frac{2}{m} r+s\right) .
$$

Si $m \notin 2 \mathbb{Z}_{>0}$, alors la paire

$$
(r, s):=\left(-\left[-\frac{1}{2} m\right], 1\right)
$$

possède la propriété exprimée a la fin de $\S 5.1$. Ici, $[x]$ pour un nombre réel $x \in \mathbb{R}$ désigne la partie entière de $x$. Donc, on déduit le lemme suivant:

Lemme 5.3. Supposons que $h=\frac{1}{24} z$ et qu'il existe $m \in \mathbb{R}_{\geq 2}$ tel que $z=z_{m}$. Si la forme $\langle\cdot, \cdot\rangle_{z, h}$ est positive semi-définie, on a $m \in 2 \mathbb{Z}_{\geq 0}$.

\subsection{Le cas $h>\frac{1}{24} z$}

Ici on suppose que $h>\frac{1}{24} z$. Alors, ils existe d'uniques nombres réels $m \in \mathbb{R}_{\geq 2}$ et $M \in \mathbb{R}_{>0}$ tels que

$$
z=z_{m}, \quad h_{m ; M}:=\frac{1}{24} z_{m}+\frac{1}{16} m M^{2} .
$$


Sous cette correspondance, on pose

$$
D(z, h):=\left\{(r, s) \in\left(\mathbb{R}_{\geq 0}\right)^{2}||-\frac{2}{m} r+s \mid \leq M\right\}
$$

Cet ensemble possède la propriété: $\operatorname{Pour}(r, s) \in \mathbb{Z}_{>0} \times\left(1+2 \mathbb{Z}_{\geq 0}\right)$,

$$
(r, s) \in D(z, h) \quad \Longleftrightarrow \quad f_{r, s}(z, h) \leq 0 .
$$

Supposons que $m \notin 2 \mathbb{Z}$ et que $D(z, h) \cap\left\{\mathbb{Z}_{>0} \times\left(1+2 \mathbb{Z}_{\geq 0}\right)\right\}=\emptyset$ pour $(z, h)=$ $\left(z_{m}, h_{m ; M}\right)$ avec $M>0$. Notons que $D\left(z_{m}, h_{m ; M^{\prime}}\right) \cap\left\{\mathbb{Z}_{>0} \times\left(1+2 \mathbb{Z}_{\geq 0}\right)\right\}=\emptyset$ pour tout $0 \leq M^{\prime} \leq M$.

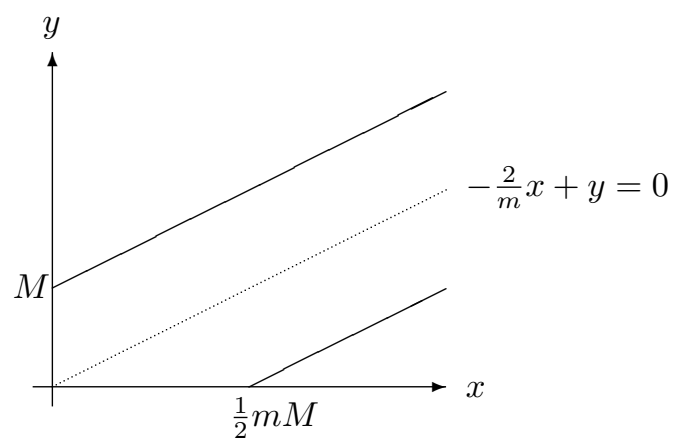

Dans ce cas, on a $\operatorname{det}(z, h)_{n}^{\tau} \neq 0$ pour tous $n \in \frac{1}{2} \mathbb{Z}_{>0}$ et $\tau \in \mathbb{Z} / 2 \mathbb{Z}$. Mais la forme $\langle\cdot, \cdot\rangle_{z, h}$ ne peut pas être positive. En effet, la forme $\langle\cdot, \cdot\rangle_{z, h} \operatorname{sur} M(z, h)$ tend vers la forme $\langle\cdot, \cdot\rangle_{z, \frac{1}{24} z}$ sur $N\left(z, \frac{1}{24} z\right)$ en passant à la limite $h \longrightarrow \frac{1}{24} z$ et le module $N\left(z, \frac{1}{24} z\right)$ n'est pas unitarisable par Lemme 5.3.

Pour le cas $m \in 2 \mathbb{Z}_{>0}$, on a $\left(\frac{1}{2} m, 1\right) \in D(z, h)$.

Donc, il suffit de considérer le cas

$$
D(z, h) \cap\left\{\mathbb{Z}_{>0} \times\left(1+2 \mathbb{Z}_{\geq 0}\right)\right\} \neq \emptyset,
$$

et on le suppose dans la discussion ci-dessous.

Posons

$$
\begin{aligned}
& r(z, h):=\min \left\{r \mid(r, s) \in D(z, h) \cap\left\{\mathbb{Z}_{>0} \times\left(1+2 \mathbb{Z}_{\geq 0}\right)\right\}\right\}, \\
& s(z, h):=\min \left\{s \mid(r, s) \in D(z, h) \cap\left\{\mathbb{Z}_{>0} \times\left(1+2 \mathbb{Z}_{\geq 0}\right)\right\}\right\} .
\end{aligned}
$$

On peut voir que $P(z, h):=(r(z, h), s(z, h)) \in D(z, h)$.

Lemme 5.4. Si $P(z, h) \in \stackrel{\circ}{D}(z, h)$ (l'intérieur de $D(z, h)$ ), la forme $\langle\cdot, \cdot\rangle_{z, h}$ n'est pas positive semi-définie.

Démonstration. Grâce à la convexité de $D(z, h)$, il n'y a qu'un point $(r, s) \in$ $D(z, h) \cap\left\{\mathbb{Z}_{>0} \times\left(1+2 \mathbb{Z}_{\geq 0}\right)\right\}$ avec la condition $r s=r(z, h) s(z, h)$. Donc, on a $\operatorname{det}(z, h)_{\frac{1}{2} r(z, h) s(z, h)}^{\tau}<0$. 
Donc, il suffit de considérer le cas $P(z, h) \in \partial D(z, h)$ (la frontière de $D(z, h)$ ). Dans ce cas, on a l'expression

$$
h=\frac{1}{24} z+\frac{1}{16} m\left(-\frac{2}{m} r(z, h)+s(z, h)\right)^{2} .
$$

On considère deux cas:

Cas $A P(z, h) \in l_{A}(m):=\left\{(r, s) \in \mathbb{R}^{2} \mid-\frac{2}{m} r+s=M\right\}$,

Cas $B \quad P(z, h) \in l_{B}(m):=\left\{(r, s) \in \mathbb{R}^{2} \mid-\frac{2}{m} r+s=-M\right\}$.

Remarquons que $P(z, h)$ ne peut pas être situé dans les autres frontières de $D(z, h)$.

Lemme 5.5. Posons $P(z, h)=\left(r_{0}, s_{0}\right) \in \partial D(z, h)$. On a $r_{0} \geq s_{0}$.

Démonstration. Supposons qu'on aie $r_{0}<s_{0}$. Puisque $m \geq 2$, le seul cas possible est le Cas $A$, mais $M \geq s_{0}-r_{0} \geq 1$, ce qui implique $\left(r_{0}, s_{0}-2\right) \in$ $D(z, h)$. Contradiction.

Pour le cas $m \in \mathbb{Z}$, on obtient

Lemme 5.6. Supposons que $m \in \mathbb{Z}_{\geq 2}$. Si $P(z, h)$ est un point de $\partial D(z, h)$, on a $r(z, h)<\frac{1}{2} m$ et $s(z, h)=1$.

Démonstration. Posons $r:=r(z, h)$ et $s:=s(z, h)$. Supposons que $s>1$.

Cas $A$ Puisque $(r, s-2) \notin D(z, h)$, on déduit que $-\frac{2}{m} r+(s-2)=M-2<-M$, c'est-à-dire, $M<1$. Par contre, l'équation $-\frac{2}{m}(r-m)+(s-2)=M$ implique $r \leq m$, c'est-à-dire, $-2 \leq-\frac{2}{m} r<0$, et on déduit que $M=$ $-\frac{2}{m} r+s \geq-\frac{2}{m} r+3 \geq 1$ d'après l'hypothèse. Contradiction.

Cas $B$ Également, on obtient $r \leq m$. Par contre, on déduit que $\frac{2}{m} r=s+M>$ $s$ qui implique $r>\frac{1}{2} s m \geq \frac{3}{2} m$ d'après l'hypothèse. Contradiction.

Donc, on a vu que $s=1$. On va montrer que $r<\frac{1}{2} m$.

Cas A La positivité de $M$ implique $r<\frac{1}{2} m$.

Cas B Par l'inégalité $r=\frac{1}{2} m(M+1)>\frac{1}{2} m \geq 1$, on doit avoir $(r-1,1) \notin$ $D(z, h)$, c'est-à-dire, $-\frac{2}{m}(r-1)+1=\frac{2}{m}-M>M$, et cette inégalité est equivalent à $m M<1$. En revanche, $m M=2 r-m \in \mathbb{Z}$ et l'hypothèse $M>0$ implique $m M \in \mathbb{Z}_{>0}$. Contradiction.

Donc, dans le reste de cet article, il suffit de montrer que le module $M(z, h)$ ne peut pas être unitarisable sous la condition $m \in \mathbb{R}_{>0} \backslash \mathbb{Z}$. Dans la suite, on suppose que $m \in \mathbb{R}_{\geq 2}$ n'est pas un nombre entier.

Lemme 5.7. Posons $\left(r_{0}, s_{0}\right):=P(z, h)$. 
1. Dans le Cas $A$, le domaine possible de $m$ est $\frac{2 r_{0}}{s_{0}}<m<\frac{2 r_{0}}{s_{0}-1}$ pour $s_{0}>1$ et $m>2 r_{0}$ pour $s_{0}=1$.

2. Dans le Cas $B$, le domaine possible de $m$ est $\frac{2 r_{0}-1}{s_{0}}<m<\frac{2 r_{0}}{s_{0}}$.

Démonstration. Dans le Cas $A$, il est nécessaire d'avoir l'inégalité $M=-\frac{2}{m} r_{0}+$ $s_{0}>0$ qui implique $m>\frac{2 r_{0}}{s_{0}}$. Puisqu'on a $\left(r_{0}, s_{0}-2\right) \notin D(z, h)$ pour $s_{0}>1$, on obtient $-\frac{2}{m} r_{0}+\left(s_{0}-2\right)=M-2<-M$, c'est-à-dire, $M<1$ qui implique $m<\frac{2 r_{0}}{s_{0}-1}$. Dans le Cas $B$, il est nécessaire d'avoir l'inégalité $-\frac{2}{m} r_{0}+s_{0}=$ $-M<0$ qui implique $m<\frac{2 r_{0}}{s_{0}}$. Puisqu'on a $\left(r_{0}-1, s_{0}\right) \notin D(z, h)$, on obtient $-\frac{2}{m}\left(r_{0}-1\right)+s_{0}=-M+\frac{2}{m}>M$, c'est-à-dire, $m M<1$ qui implique $m>$ $\frac{2 r_{0}-1}{s_{0}}$.

Sous l'hypothèse d'unitarisabilité de $M(z, h)$, on a la restriction suivante sur $s_{0}$.

Lemme 5.8. Si $M(z, h)$ est unitarisable, on a $s_{0}=1$.

Démonstration. Supposons que $s_{0}>1$ et $M(z, h)$ est unitarisable pour un certain $m^{\prime} \in \mathbb{R}_{\geq 2}$. Alors, les nombres entiers $r_{0}$ et $s_{0}$ sont premiers entre eux. En effet, sinon, il existe $N \in \mathbb{Z}_{>1}$ et $\left(r_{1}, s_{1}\right) \in \mathbb{Z}_{>0} \times\left(1+2 \mathbb{Z}_{\geq 0}\right)$ tels que $\left(r_{0}, s_{0}\right)=N\left(r_{1}, s_{1}\right)$, et on a $\left(r_{1}, s_{1}\right) \in D(z, h)$ ce qui ne se peut.

Pour le Cas $A$, le Lemme 5.7 implque $\frac{2 r_{0}}{s_{0}}<m^{\prime}<\frac{2 r_{0}}{s_{0}-1}$. D'après l'hypothèse, on a

$$
\operatorname{det}(z, h)_{n}^{\tau}>0 \quad \forall n<\frac{1}{2} r_{0} s_{0}, \quad \frac{2 r_{0}}{s_{0}}<\forall m \leq m^{\prime} .
$$

Considérons la limite $m \rightarrow m_{0}:=\frac{2 r_{0}}{s_{0}}$. Le poids $(z, h)$ tend vers $\left(z_{m_{0}}, \frac{1}{24} z_{m_{0}}\right)$ par cette limite. En particulier, on a $r\left(z_{m_{0}}\right)=\left[\frac{r_{0}}{s_{0}}\right]+1$ et $s\left(z_{m_{0}}\right)=1$. Puisqu'on a $\left(r_{0}, s_{0}\right)=1$, l'hypothèse $s_{0}>1$ implique $\operatorname{det}\left(z_{m_{0}}, \frac{1}{24} z_{m_{0}}\right)_{\frac{1}{2} r\left(z_{m_{0}}\right)}^{\tau}<0$. Par contre, la même hypothèse implique

$$
\frac{1}{2} r\left(z_{m_{0}}\right)=\frac{1}{2}\left(\left[\frac{r_{0}}{s_{0}}\right]+1\right)<\frac{1}{2} r_{0} s_{0}
$$

ce qui contredit (6). Pour le Cas $B$, le Lemme 5.7 implque $\frac{2 r_{0}-1}{s_{0}}<m^{\prime}<\frac{2 r_{0}}{s_{0}}$. La démonstration est analogue au Cas $A$ en passant à la limite $m \rightarrow m_{0}$.

Donc, on suppose que $P(z, h)=\left(r_{0}, 1\right) \in \partial D(z, h)$. Dans ce cas, le Lemme 5.7 implique $m>2 r_{0}-1$. Supposons que $M(z, h)$ est unitarisable pour certain $m_{0} \in \mathbb{R}_{>2 r_{0}-1} \backslash \mathbb{Z}$.

Si $2 r_{0}-1<m_{0}<2 r_{0}+1$, il est facile de voir que $\overline{\operatorname{det}}(z, h)_{\frac{1}{2}\left(r_{0}+1\right)}<0$ grâce à Théorème 4.2. Si $m_{0}>2 r_{0}+1$, il est facile de voir que $\overline{\operatorname{det}}(z, h)_{\frac{1}{2}\left(\left[m_{0}\right]+1\right)}^{\tau}<0$ grâce au Théorème 4.2. Contradiction. 


\section{References}

[BFK] W. Boucher, D. Friedan et A. Kent, Determinant Formulae and Unitarity for the $N=2$ Superconformal Algebras in Two Dimensions or Exact Results on String Compactification, Phys. Lett. B 172, (1986), $316-322$.

[CP] V. Chari et A. Pressley, Unitary representations of the Virasoro algebra and a conjecture of Kac, Compos. Math. 67, (1988), 315-342.

[FQS1] D. Friedan, Z. Qiu et S. Shenker, Conformal Invariance, Unitarity, and Critical Exponents in Two Dimensions, Phys. Rev. Lett. 52, (1984), $1575-1578$.

[FQS2] D. Friedan, Z. Qiu et S. Shenker, Superconformal Invariance in Two Dimensions and Tricritical Ising Model, Phys. Lett. B 151, (1985), $37-43$.

[GKO] P. Goddard, A. Kent et D. Olive, Unitary Representations of the Virasoro and Super-Virasoro Algebras, Comm. Math. Phys. 103, (1986), $105-119$.

[IK] K. Iohara et Y. Koga, Representation theory of $N=2$ super Virasoro algebra: twisted sector, Jour. Funct. Anal. 214, (2004), 450-518.

[K] V. G. Kac, Infinite dimensional Lie algebras, $3^{\text {rd. }}$ ed., Cambr. Univ. Press, 1991.

[L] R. P. Langlands, On unitary representations of the Virasoro algebra, in Infinite dimensional Lie algebras and their applications, S. Kass (ed.), Singapore, World Scientific, 1988.

[LW] Lepowsky J. et R. L. Wilson, A new family of algebras underlying the Rogers-Ramanujan identities and generalizations, Proc. Natl. Acad. Sci. USA 78, (1981), 7254-7258.

[RS] Rittenberg V. et Schwimmer A., A Connection between the Twisted $N=2$ Superconformal and $S U(2)$ Kac-Moody Algebras, Phys. Lett. B 195, (1987), 135-138.

[S] F. Sauvageot, Représentations unitaires des super-algèbres de Ramond et de Neveu-Schwartz, Comm. Math. Phys. 121, (1989), 639-657.

[ZF $]$ A. B. Zamolodchikov et V. A. Fateev, Disorder fields in twodimensional conformal quantum-field theory and $N=2$ extended supersymmetry, Zh. Eksp. Teor. Fiz. 90, (1986), 1553-1566. 